\title{
EFFECTS OF CYBERKNIFE RADIOTHERAPY TREATMENT OF PITUITARY ADENOMAS
}

S. Puglisi ${ }^{1}$, O.R. Cotta ${ }^{1}$, A. Conti ${ }^{2}$, A. Pontoriero ${ }^{3}$, E. Messina ${ }^{1}$, A. Albani ${ }^{1}$, F. Ferrau'1, M. Ragonese ${ }^{1}$, M.L. Torre ${ }^{1}$, F. Angileri ${ }^{2}$, S. Cannavo' ${ }^{1}$

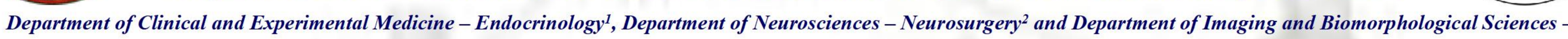

Radiotherapy ${ }^{3}$. University of Messina, Messina, Italy

INTRODUCTION

$>$ CyberKnife (CK), a LINAC-based robotic device for frameless stereotactic surgery is an emerging treatment for pituitary tumours (PT) resistant to other therapies.

\section{PATIENTS AND METHODS}

$>$ We report long-term CK effect on endocrine function and tumour volume in 20 PT patients (10M/10F, mean age $57.5213 .97 \mathrm{yrs})$.

> Twelve patients harbored a non functioning adenoma (NFPA), 2 an ACTH, 5 a GH (1 case of TSH co-secretion) and one a PRL-secreting PT.

$>$ Before CK 9 patients had normal while 11 presented an impaired pituitary function (4 cases of isolated pituitary deficiency, 5 multiple pituitary deficiencies and 2 panhypopituitary, Fig. 1).

Patients were treated using conventional CK (Cyber-Knife ${ }^{\circledR}$, Accuray, Inc., Sunnyvale, CA) multisession radiosurgery schemes (1-5 fractions); mean volume of the treated lesions was of $8.8711 .3 \mathrm{cc}$; mean marginal dose was $214.9 \mathrm{~Gy}$, mean prescription isodose line $72.95 \quad 5.3 \%$ and mean coverage $96.1 \quad 1.37 \%$.

CK was used as first line treatment in 3 cases (2 NFPA and $1 \mathrm{GH} / \mathrm{TSH}$ secreting adenoma) and for treating residual pituitary tumours in the other 17 cases.

The mean follow-up period was 24.1720 .53 months (range 3-112 months).

\section{RESULTS}

MRI : demonstrated tumour shrinkage in 55\% of patients: in 3 NFPA cases tumour was no longer visible ( 1 case of first line treatment) while 8 cases presented significant tumour shrinkage (6 NFPA patients, $1 \mathrm{GH}$ and $1 \mathrm{ACTH}$ secreting PT). In 6 cases no volumetric variations were registered; tumour increase was evident only in 3 cases: 1 NFPA, 1 aggressive GH and one aggressive PRL secreting PT (Fig. 2).

> Pituitary function impairment occurred in 4 of the 9 patients with previous normal pituitary function who developed isolated deficiency in 2 cases and multiple deficiencies in the other 2 .

$>$ Among 6 patients with previously multiple or isolated hypopituitarism, 5 became panhypopituitary and 1 developed a new deficit (Fig. 3).

One acromegaly patient, previously resistant to medical treatment normalized IGF-I levels.

\begin{tabular}{|ccccc|}
\hline Patient & Age (yrs) & Gender & $\begin{array}{c}\text { Tumour } \\
\text { Type }\end{array}$ & $\begin{array}{c}\text { Tumou } \\
\text { Size }\end{array}$ \\
\hline 1 & 56 & M & NFPA & Macro \\
\hline 2 & 46 & F & NFPA & Macro \\
\hline 3 & 42 & F & NFPA & Macro \\
\hline 4 & 79 & F & NFPA & Macro \\
\hline 5 & 57 & M & NFPA & Macro \\
\hline 6 & 43 & F & NFPA & Macro \\
\hline 7 & 69 & M & NFPA & Macro \\
\hline 8 & 75 & M & NFPA & Macro \\
\hline 9 & 51 & F & NFPA & Macro \\
\hline 10 & 54 & F & NFPA & Macro \\
\hline 11 & 75 & F & NFPA & Macro \\
\hline 12 & 69 & F & NFPA & Macro \\
\hline 13 & 36 & M & GH & Macro \\
\hline 14 & 59 & F & GH & Macro \\
\hline 15 & 65 & M & GH/TSH & Macro \\
\hline 16 & 49 & M & PRL & Macro \\
\hline 17 & 75 & F & GH & Macro \\
\hline 18 & 49 & M & ACTH & Macro \\
\hline 19 & 35 & M & ACTH & Macro \\
\hline 20 & 65 & M & GH & Macro \\
\hline
\end{tabular}

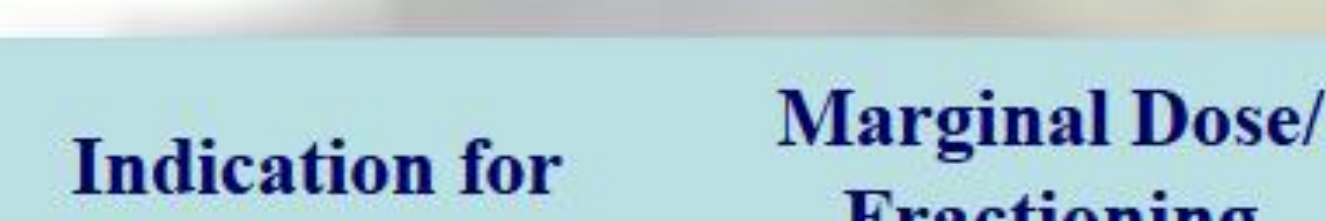

Indication for
CK treatment

Fractioning Isodose Coverage (Gy/fr)

$\uparrow \varnothing+$ ASA 4

$22 / 4$

NO

TNS

TNS (2)

$\uparrow \varnothing$

$\uparrow \varnothing$

$\uparrow \varnothing$

$\uparrow \varnothing$

$\uparrow \varnothing$

$\uparrow \varnothing$

$\uparrow \varnothing+$ ASA 4

$\uparrow \varnothing$

$\uparrow \varnothing$

$\uparrow \varnothing$

$\uparrow \varnothing$

SSa+PEG Resistance

SSa e PEG Intolerance

+ ASA 4

$\uparrow \emptyset+\mathrm{CAB}$ Resistance

SSa+PEG Resistance

$\uparrow \varnothing$

$\uparrow \varnothing$

$\uparrow \varnothing$

$\begin{array}{lll}23 / 5 & 68 & 97\end{array}$

$\begin{array}{lll}24 / 3 & 75 \quad 97\end{array}$

$30 / 5 \quad 75 \quad 93$

$27.5 / 5 \quad 74 \quad 96$
SSa+PEG Resistance

$25 / 5 \quad 75 \quad 95$

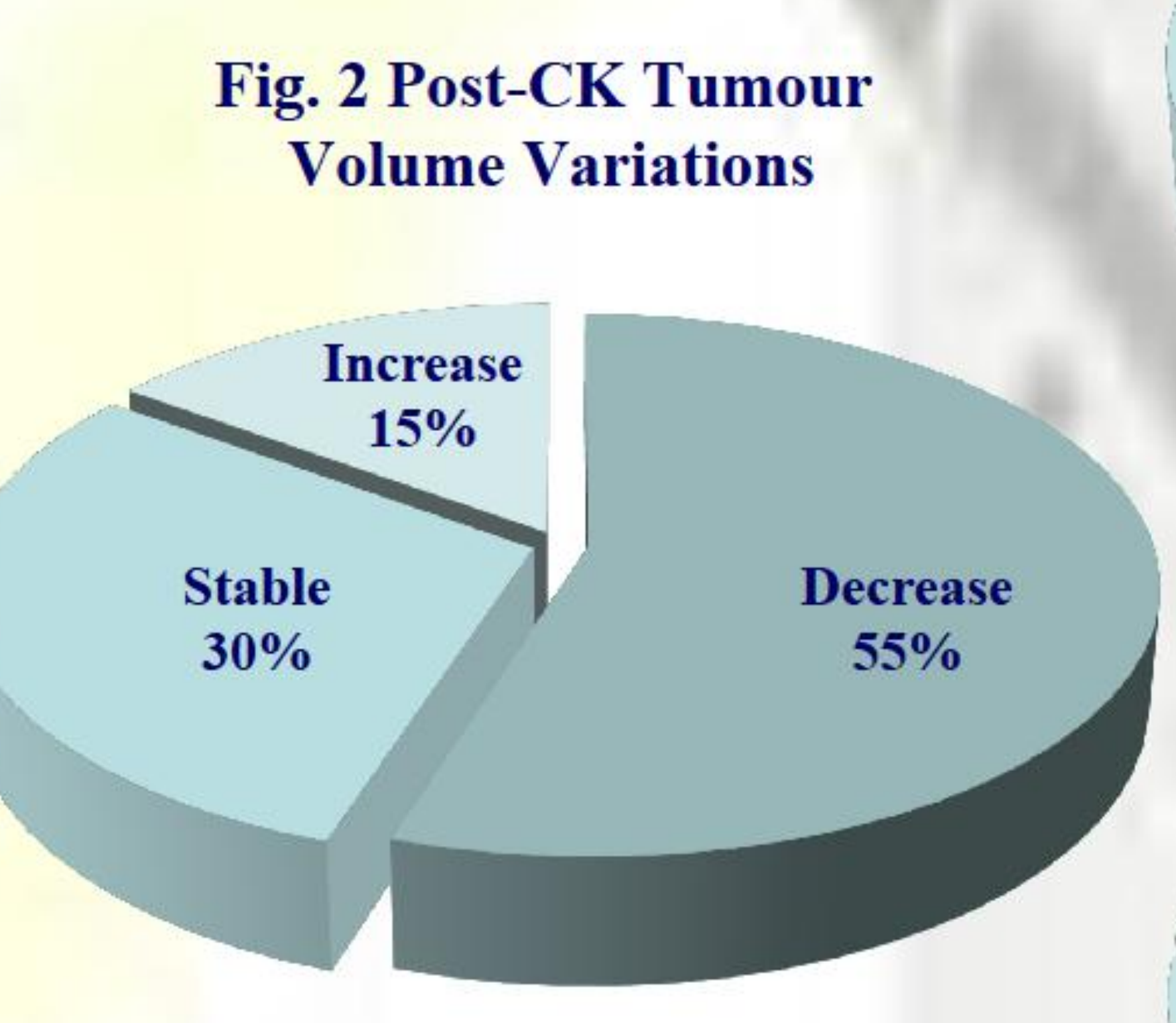

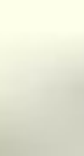

Fig. 1 Pre-CK Pituitary Function
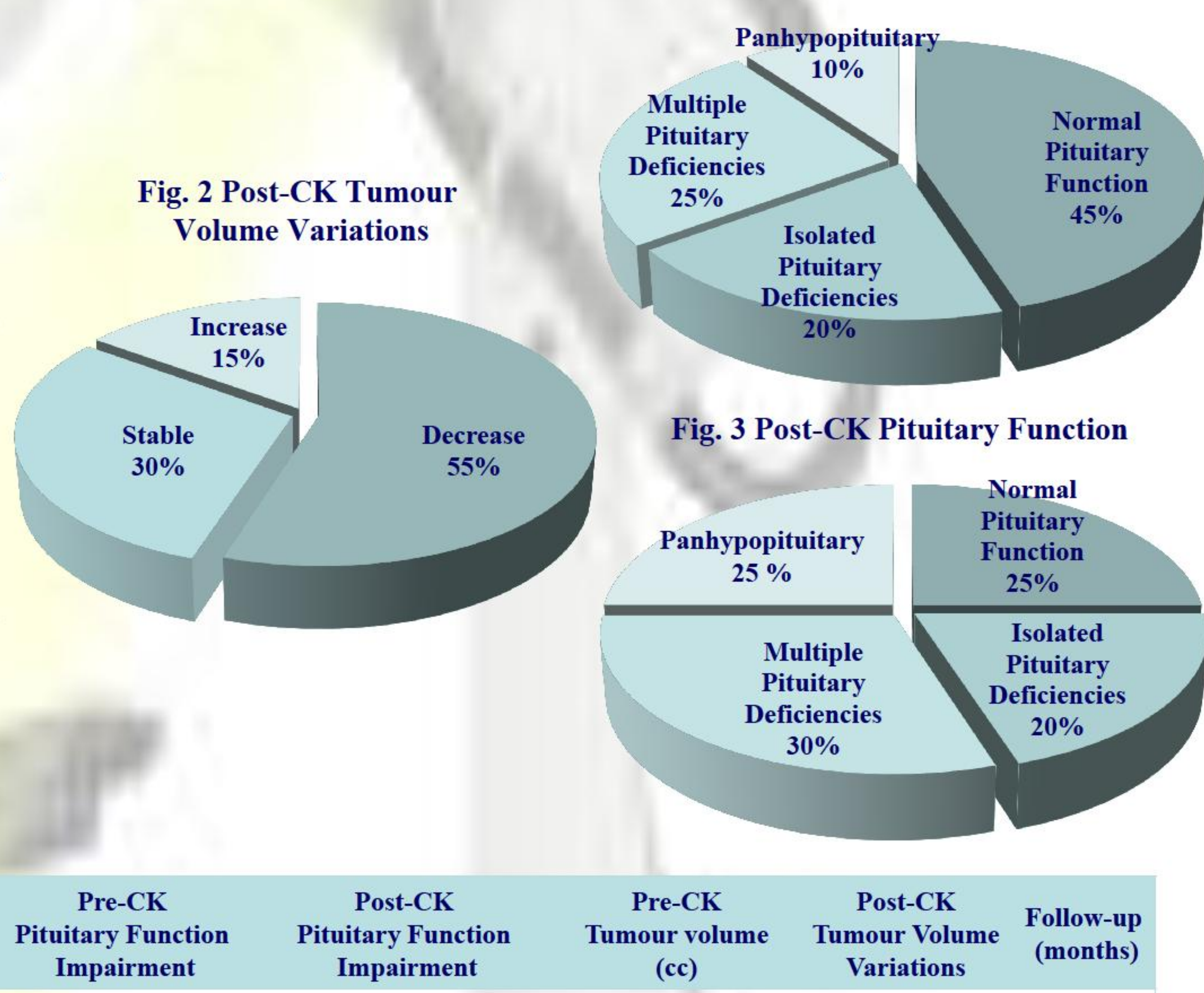

NO

TSH-GH-LH/FSH PANHYPOPITUITARY

TSH-GH PANHYPOPITUITARY

TSH-GH-LH/FSH PANHYPOPITUITARY

TSH-GH-LH/FSH PANHYPOPITUITARY

NO NO

$\mathrm{GH}$

NO

NO

NO

NO

LH/FSH

LH/FSH

NO

NO

GH

GH-LH/FSH

$\mathrm{GH}$

TSH-GH-LH/FSH

NO

GH-LH/FSH

LH/FSH

LH/FSH

NO

NO

$$
\begin{aligned}
& \text { GH } \\
& \text { NO }
\end{aligned}
$$

TSH-GH-LH/FSH

GH-LH/FSH

TSH-LH/FSH-ACTH

Table 1: TNS trans-naso-sfenoidal, $\mathrm{CT}$ transcranial, SSa somatostatin analogs, $\mathrm{CAB}$ cabergoline, PEG Pegvisomant, $\uparrow$ increase, $\downarrow$ decrease,
CONCLUSIONS

CK treatment for PT is safe and effective, ceasing tumour growth in $85 \%$, and inducing tumour shrinkage in $55 \%$ of cases. Nevertheless, impairment of pituitary

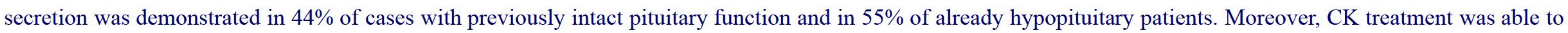
obtain disease control of resistant acromeglay. 